\title{
Follow-up and Diagnose COVID-19 Using Deep Learning Technique
}

\author{
Bakhtyar Ahmed Mohammed ${ }^{1,2, *}$, Muzhir Shaban Al-Ani ${ }^{2,3}$ \\ ${ }^{1}$ University of Human Development, College of Science and Technology, Department of Computer Science, Sulaymaniyah, KRG, Iraq \\ ${ }^{2}$ University of Sulaimani, College of Science, Department of Computer, Sulaymaniyah, KRG, Iraq \\ ${ }^{3}$ University of Human Development, College of Science and Technology, Department of Information Technology, Sulaymaniyah, KRG, \\ Iraq
}

\begin{tabular}{l} 
A R T I C L E I N F O \\
\hline Article history: \\
Received: 12 January, 2021 \\
Accepted: 01 April, 2021 \\
Online: 22 April, 2021
\end{tabular}

Keywords:

Follow-up

Diagnosis

COVID-19 Pneumonia

Deep Learning

Deep convolutional neural network

A B S T R A C T
In recent days, the fast growth of populations leading to an increase in medically
complicated cases, especially fast spread viral cases around the world. These phenomena
increased demand on auto-diagnose systems to speed up the diagnosis process and reduce
human contacts, especially for the COVID-19 pandemic using deep learning (DL). DL
methods can successfully carry out these complicated works. A Deep Convolutional Neural
Network (Deep CNN) is the most appropriate model for the medical image diagnosis process
among DL techniques. This study focuses on follow-up and diagnosis of COVID-19
pneumonia cases. Deep CNN model can learn the chest computed tomography (CT) features
properly synchronizing with the training options that involve the optimizer, number of
epochs, and learning rate to get optimal accuracy with the lowest error rate. The auto
diagnosis process aims to follow-up and diagnosis COVID-19 pneumonia and illuminate it
from Streptococcus pneumonia and normal chest. Executed the present study on were 840
CT images of 24 patients from the Radiopedia database. Computed tomography (CT) is the
best modality to visualize lung diseases, which own enough positions to interpret everything
inside lung anatomy. Deep CNN model owns of enough layers and enable the model to
extract and learn pneumonia features from the training set images. This process applied on
MATLAB software. The model's result exhibits that the proposed deep CNN approach had
an accuracy level of $99.37 \%$.

\section{Introduction}

In 2019, new type of coronavirus found in china for first time which known as COVID-19 [1]. It is a type of corona virus family, which causes of pneumonia in severe situations [2]. With increasing the COVID-19 cases around the world, demand of advanced computer-aided lung CT diagnosis systems to followup and surveillance severe cases increased [1], [3]. Similar pulmonary syndrome shows same features between various Corona virus types, such as COVID-19, Severe Acute Respiratory Syndrome (SARS), and Middle East Respiratory Syndrome (MERS) [2]. The main benefit of DL is speeding up the diagnosis process compare to manually diagnosis. It is dominant field among all machine-learning techniques. It is too beneficial to reduce misdiagnosis and false positives (FP). Deep convolutional

\footnotetext{
*Corresponding Author: Bakhtyar Ahmed Mohammed, Email:

bakhtyar.mohammed@uhd.edu.iq

Www.astesj.com
}

https://dx.doi.org/10.25046/aj0602111 neural network (deep CNN) or ConvNet in DL can learn such complex features to diagnosis process. In addition, it can differentiate in histologically similar tissues more accurate than manually diagnosis [4]. DL involves the processes of feature extraction and classification together for medical image diagnosis process [5]. It can detect ground glass opacity (GGO) and conjunction spot places as significance features [2]. In severe situations, this viral contagious varies to a type of pneumonia. Sometimes causes to multi-organ failure. CT modality is the best technique to follow-up the novel COVID-19 pneumonia (NCP) cases. It can exhibit significant features of COVID-19 pneumonia [6]. CT images of the types of corona virus family have similar features in pneumonia situations [3]. Chest CT features of COVID-19 are different from other types of viral pneumonia, such as; seasonal flu pneumonia or Streptococcus pneumonia [7]. Huge number of COVID-19 cases in everywhere made the medical scientists and researchers think about various ways to 
diagnose suspected cases. Chest CT scan is one way to diagnose COVID-19, which causes medication staffs, use it to follow-up severe cases [2], [8]. Collecting datasets is challenging processes that need a lot of time to solve of this problem is getting AI-driven tool, which invented to work parallel with real-time diagnosis in hospitals to speed up the COVID-19 diagnosis process [9]. Deep CNN compatible with scalability of datasets automatically, such as highly representative, layered hierarchical image features from sufficient training data [10]. In back-propagation process, deep learning enables methods to learn features which relies on the derivative of loss function as gradient descent [11]- [13]. Features of CT images for chest diagnosis, such as ground glass opacity (GGO) to detect COVID-19 pneumonia cases, made it useful in training process in improving computer-aided methods as a fast process. In addition, it aids the clinicians especially in the diagnosis of COVID-19 infection cases [1].

Usually this severe viral contagious confirms by reversetranscription polymerase chain reaction (RT-PCR). Sensitivity of RT-PCR, which is not properly finding COVID-19 because it cannot activate the virus antigen in early stages of the disease. Because of this reason, CT modality use as an effective way to early screening and follow-up the virus impacts on patients' lungs [3]. Availability of fast and accurate method to diagnose COVID19 pneumonia is cause to effective treatment properly. This approach implemented appropriate deep CNN method to recognize COVID-19 pneumonia among three classes of viral pneumonia, bacterial pneumonia and normal lung. The training method sat to optimum situation.

\section{Background}

COVID-19 is the seventh member of corona virus family that infect human and animal. Similarity gene of the COVID-19 with SARS is above $70 \%$ and identically of the virus with corona virus of bats is $96 \%$ [14]. In 2020, Julian Liu exhibited how symptomatic and asymptomatic COVID-19 diagnosed for first time using chest CT via similar features with old versions of corona virus family types, such as; SARS and MERS. Generally, three clinical manifestations rely on to diagnose COVID-19, such as; symptoms, PCR nuclei test, and CT scan. However, for suspicious cases, at the first stage confirming by $\mathrm{CT}$ is effective, reliable, and accessibility aid of diagnosing the suspicious cases. He showed the importance of artificial intelligence (AI) device of Infer vision Inferred CT Pneumonia AI of software support improvements in radiology branch especially COVID-19 pneumonia diagnose process using AI. It is safe contact-free software to recognize COVID-19 pneumonia features using DL in short period of time [15].

Clinicians distributed COVID-19 patients into four groups, such as; mild, moderate, severe, and crucial types according to their lung CTs to follow-up the patients who classified accurately especially in severe conditions using deep learning [16]. In 2020, Dimpy Varshni determined an optimal CNN model for binary classification of pneumonia patients which known as Streptococcus pneumonia and normal cases based on their chest $\mathrm{x}$-rays. This system is too beneficial especially in remote areas [17]. Also, Lin Li developed 3D DL framework to detect COVID19. He used segmentation and detection for the classes of COVID19 , community acquired pneumonia (CAP), and non-pneumonia.
First process starts by pre-processing to extract lung regions, as regions of interest (ROI) using U-net segmentation method. Second step involve ResNet-50 architecture to extract 2D, and 3D representative features depend on $\mathrm{CT}$ slices to detect the classes [3].

In 2020, Song Ying proposed a useful developed computer aided method for clinicians to fast diagnoses of the COVID-19 infectious cases. The method is DRE-net as new DL technique. It can localize main lesion features like GGO in 30 seconds using Tianhe- 2 super computer. This device can perform southern tasks simultaneously with the online available server with accuracy $94 \%$ [1].

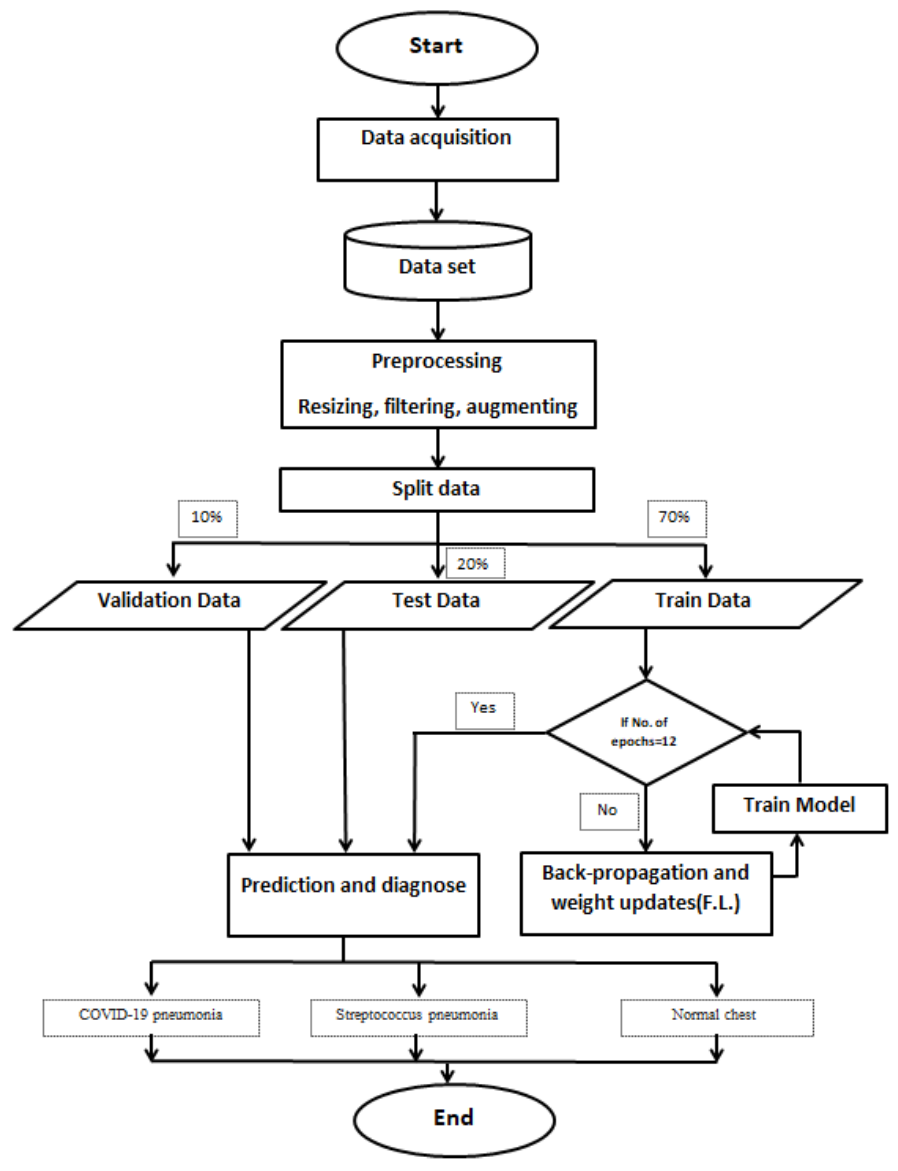

Figure 1: Basic Flow Chart

In addition, Xiaowei Xu proposed a DL system for early screening COVID-19 according to three classes of COVID-19 pneumonia, Influenza-A viral pneumonia, a normal chest via their CT images using DL. However, the problem of real-time RT-PCR to diagnose RNA of the virus in sputum and nasopharyngeal swap is too low rate of positive cases in early stages of COVID-19, but CT scan exhibits the features of COVID-19 pneumonia [7]. In spite of, Chuansheng Zheng proposed a pre-trained UNet for lung region segmentation and weakly supervised deep learning model. It is 3D deep neural network to predict the probability of COVID19 infections using 499 CT volumes without need for annotating the lesions for training with accuracy $95.9 \%$ [18].

In 2020, Ali Narin proposed a quick automatic detection system rely on five pre-trained CNN models of ResNet50 with highest accuracy which is $96.1 \%$, ResNet101, ResNet152, 
InceptionV3 and Inception-ResNetV2 according to the classes of COVID-19, normal (healthy), viral pneumonia and bacterial pneumonia [19]. Furthermore, Mohamed Loey proposed a generative adversarial network (GAN) with deep transfer to detect COVID-19 in early stages that increase recovery possibilities. The study relied on 307 chest $\mathrm{x}$-ray images according to four classes of COVID-19, normal chest, bacterial pneumonia, and viral pneumonia depend on the models of AlexNet, GoogleNet and ResNet18 [20].

The COVID-19 auto-diagnose system relies on two important factors which are materials and methodology. Materials compose of the dataset of chest CT raw data and hardware parts. Methodology involves the steps of the proposed system. The current study applied on the CT chest images dataset from Radiopedia in 2020 using deep CNN in order to follow-up and auto-diagnosis COVID-19 pneumonia cases. The proposed approach concentrated on using suitable deep CNN architecture, and the training process options properly with the dataset and necessary tools.

\section{Materials and Methodology}

It involves every imperative things to implement the system, such as; the labeled dataset to train, test and validate the deep $\mathrm{CNN}$ model, suitable platform to run the deep CNN codes on which uses MATLAB and hardware resource like; graphical processing unit (GPU). Preparing the necessary things is the most imperative processes to implement the auto-system via optimum way.

\subsection{Methodology}

It consists of the practice part of auto-diagnosis processes according to the task priorities. The auto-diagnose system involve some operations, as illustrated in Fig. 1. As indicated in the figure, the system involves the steps of; CT chest data acquisition, preprocessing, distributing the dataset, data augmentation, creating deep CNN, selecting training options, predict and diagnosis.

- CT chest data acquisition: It is an essential process to perform the process accurately because supervising learns models learn from the labeled dataset. It is the primary handle to form a good dataset in any case, which is challenging. Medical image modalities are the foremost imperative components to urge the fitting dataset. The examined depended on utilizing computed tomography (CT) picture methodology. It is the foremost prevailing one to display the lungs. The dataset composed of $840 \mathrm{CT}$ images obtained from Radiopedia database of 24 patients for the classes of COVID-19 pneumonia, Streptococcus pneumonia and normal chest. (See Fig. 2).

- Pre-processing: It composed of three processes, which are; resizing, de-noising, and CT image data augmenting. CT chest images in situations of pneumonia diagnosis have some problems, which cause of lessening of diagnosis accuracy, such as; noise, missing values, and inhomogeneous region of interest (ROI). In this regard, pre-processing processes solve these processes of the collected dataset. First pre-processing process performs by standardizing the dataset image sizes into an appropriate size, which set to $512 * 512 * 1$. Second pre- processing process is filtering. This process performed using median filter for de-noising and preserves edges. It used to solve the problem of missing values. Data augmentation enables the system to interpret images from positions of rotation, scaling, reflection, translation, and cropping. In addition, it used instead of solving the lack of the dataset and increasing capacity to analyze maximum features.

- Distributing the dataset: It is partitioning process for each of the classes into $70 \%$ for training set, $20 \%$ for testing set, and $10 \%$ for validation sets. MATLAB tool has ready function to split each of labeled classes according to their rations.

- Creating Deep CNN: It is another imperative step in the process because it determines which architecture is suitable to solve the case. Deep CNN enables the auto-diagnosis system to extract GGO features properly with the deep CNN architecture and training process parameters, such as; number of epochs, optimizer, and learning rate. These two parts have the most influences on the process accuracy. Architecture of the deep CNN involves the feature extraction and classification layers. Feature extraction layers composed of image input layer, convolution 2D layer, rectified linear unit (ReLU) layer, batch normalization layer, max pooling 2D Layer. This process repeats until the final pooled feature maps produce. Classification layers composed of fully connected layer (FCN), SoftMax, classification layers. Some parameters have the most influences on accuracy of the proposed approach, such as; organizations of the deep CNN method, datasets, optimizers, learning rate, and the number of epochs.

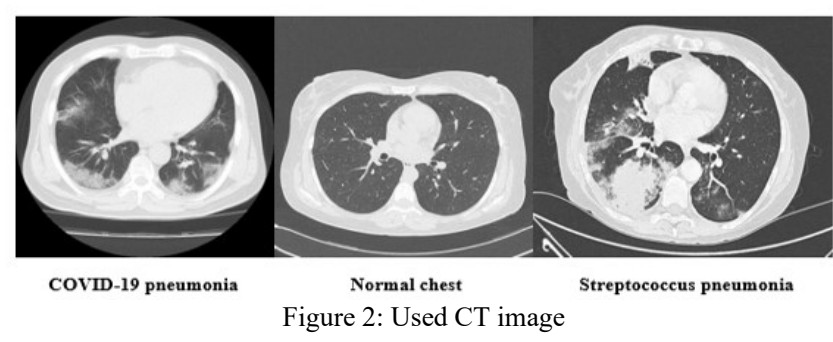

The proposed deep CNN model utilizes based on the mechanism of the CNN. The components of the deep CNN explained below:

Convolution layer: it is linear process between input images and impulse response. Impulse response knew as mask or filter. The processes performed using a 2-dimensional (2D) convolution, as shown in Equation 1. It performed by convolving both horizontal and vertical directions in 2D spatial domain.

$$
Z_{x, y}=\sum_{l} \sum_{k} W_{l, k} a_{x-l, y-k}+b=W \otimes a_{x, y}+b
$$

where $\left(a_{x, y}\right)$ is $2 d$ input image values, (w) is kernel or weight, (b) is bias, $(\mathrm{x}, \mathrm{y})$ extends the dimensions of the input, and $\mathrm{l}, \mathrm{k}$ extends the dimensions of the kernel.

ReLU layer: it is a non-linear relationship of convolution result process. Its primary utilization is using for hidden layers of the neural network. This function only lets the maximum values pass during the front propagation. As shown in Equation 2. 


$$
W_{l, k}=h\left(Z_{x, y}\right)
$$

Batch normalization layer: This layer is responsible for distributing each class of the $\mathrm{CT}$ images dataset into smaller batch sizes in order to run the program properly with the processing unit. It is a procedure for preparing as well profound neural systems and standardizing the inputs to a layer for each mini-batch. The algorithm distributed the classes by mini batch sizes of 32, which cause decline run-time.

Max pooling: It discounts includes sizes indeed, when zero cushioning increments include more extended interior the image. Pooling may be a way to require huge pictures and shrivel them down whereas protecting the foremost critical data in them, which declare to as highlights. After all these forms, the highlights arranged by means of the pooled highlight, maps. This process continues until the final max-pooling layer in the last epochs.

Include extraction in each directed learning strategy depends on datasets. In this respect, DL strategies are cleverly strategies to memorize highlights from the preparing set.

Fully connected ( $F C N$ ) layer: it is the first layer of classification process. The image values arrange in this layer in the form of a vector. FCN is the advanced level of filtered images. The images values interpret vertically, and it translates to prediction and vote.

SoftMax layer: It is an activation function that typically applied on the output of the final layer.

Classification Layer: It is the last layer inside the deep CNN architecture, which is responsible for selecting the number of the classes.

After any forward propagation, a result produces which called actual result. In addition, every process has target result. These two results determine error total. As shown in Equation 3.

$$
\mathrm{E}_{\text {total }}=\sum 1 / 2\left(\text { Output }_{\text {target- }} \text {-Output }{ }_{\text {actual }}\right)^{2}
$$

After each of the forward propagation process, the backward propagation process comes to adjust the weight values to make the system train to learn the features to increase the accuracy, (See Equation 4).

$$
\delta_{x, y}(l)=\frac{\partial E}{\partial Z_{x, y}(l)}=\sum_{u} \sum_{v} \frac{\partial E}{\partial Z_{u, v}(l+1)} \frac{\partial Z_{u, v}(l+1)}{\partial Z_{x, y}(l)}
$$

The processes of forward and backward propagation continue from under-fitting to optimal means the accuracy arrives to the highest plateau, which is lowest error rate. As shown in Figure 3.

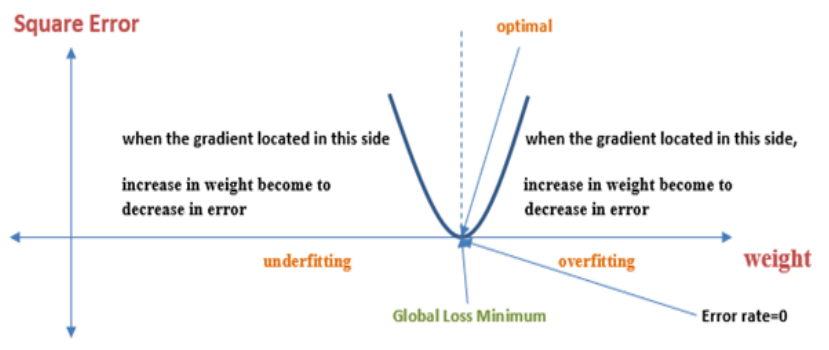

Figure 3: Optimum case in Gradient Descent
This study relied on multi-classification to classify three types of chest situations relate to COVID-19 pneumonia diagnosis. It arranged in three classes, including COVID-19 pneumonia, Streptococcus pneumonia and normal chest with accuracy of $99.37 \%$.

Selecting training options: Training options are the most effective factors to increase the learning capacity. It controls the learning process appropriately. The learning process or training performed through back-propagation process. The training option of auto-diagnose the process involve such effective parameters as; stochastic gradient descent with momentum (SGDM), epoch numbers, and learning rate. Gradient descent method is responsible for adapting the parameters to decrease error rate gradually according to epoch numbers. SGDM optimizer is an important parameter for the training process. It is an adaptive learning rate method, which calculates the derivative of total error with output of each process via back-propagation and updates their weights. The optimizer determines next place where it goes on the curve. It calculates the individual learning rates to various parameters with the initial learn the rate of 0.001 . These factors are important and effective to the training process because they enable the method to get maximum accuracy and the lowest error rate. It solves according to the equation 5 .

$$
\mathrm{M}=\mathrm{a}-\mathrm{N} \Delta \mathrm{f}(\mathrm{a})
$$

while $\mathrm{M}$; is next value, a; is current value, $\mathrm{N}$; is the learning rate which is a hyper-parameter, $\Delta \mathrm{f}$ (a) is the path of the steepest descent. This process determines the path of optimal weights the formula tells the next position where the optimal way goes, which is the direction of the steepest descent.

- Diagnose and detection: This process indicates true positive and negative situations of the classes relate to the decision making about the pneumonia cases. The proposed method can recognize the new image features accurately based on the trained features.

In this study, the dataset labeled according to three classes, such as; COVID-19 pneumonia, Streptococcus pneumonia and normal chest. The chest $\mathrm{CT}$ image dataset collected from Radiopedia of 840 lung CT images of 24 cases with both axial and coronal states. The dataset included the images, which own of the most significance features. Then the images pass through preprocessing step using image resizing, filtration and augmentation to decrease noise and enhance the images quality and increase readability. The images resized to size of $512 * 512 * 1$. Median filter used to remove noise problem of missing values and compensating missing values without removing original image pixels and the images augmented to increase feature extraction capacity.

After that, the images feed to the Deep CNN method to recognize and diagnose COVID-19 pneumonia and compared it to; Streptococcus pneumonia and Normal chest according to tested image features. DL is capable to learn complicated features, as; ground glass opacity (GGO) of COVID-19 pneumonia features which reveal them by CT images. Every methods of CNN model pass through the processes inside the deep CNN model for feature extraction and classification purposes. 


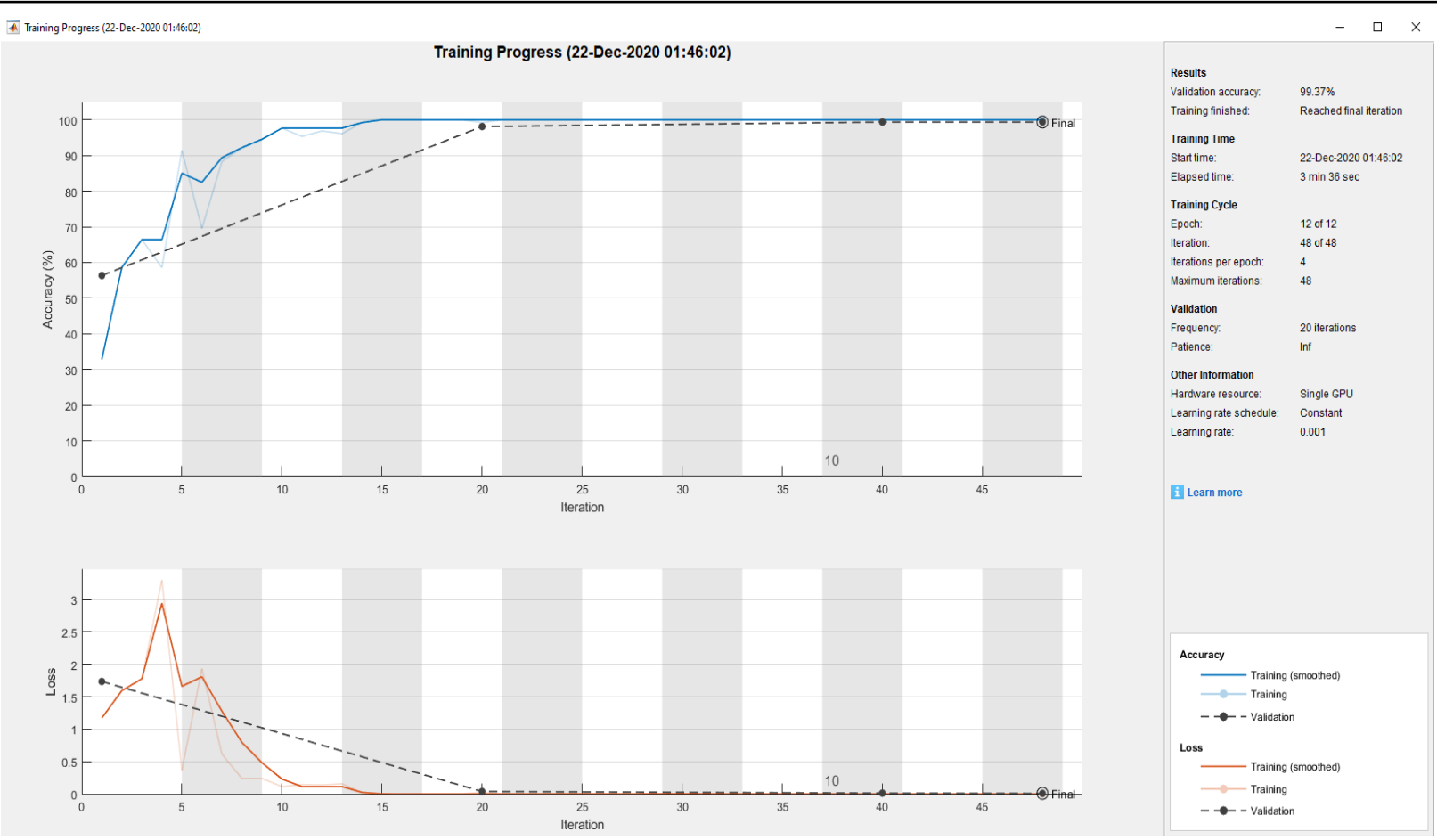

Figure 4: The process implemented on MATLAB

Organization of the Deep CNN is the most important factor in the diagnosis process. Because it determines which architecture, appropriate to solve the case. The architecture of the proposed model involves multi-hidden layers for feature extraction process. This process relies on multi-layer perceptron (MLP). While the digital image values pass through the deep CNN method. The transmission of image values in neural network depends on two processes according to how the adjacent nodes transmit image values to next layer node. The image values go through the convolution layer by convolving the image values and weights, and pooling and ReLU layers. These layers perform the process of feature learning. Weights represent filters. The input image passes through forward and backward propagation in an epoch, while the connections between adjacent layer nodes are weighted.

The weights essentially are highest similar feature values, which own of the most similarity. According to these similar features, the deep CNN method can select the most significant features. The model extracts the homogeneity of the images based on the training set labeled classes.

Goal of the back-propagation process is to update each of the weights of the nodes or neurons from the output layer to the input layer according to the number of epochs in the network in order to the actual output is closer to the target output. Feature learning or feature extraction learns in the backward process according to the number of epochs and iterations, Batch size, learning rate and optimizer. Number of epochs and iterations are the important factors to enhance the performance of the operation. One epoch means one time the process completes forward and backward propagation according to stochastic gradient descent method. As the number of epochs increases, a greater number of times the weights changed in the neural network and the curve go from under-fitting to optimal then to over-fitting according to the repeating number of epochs. Batch size determines how many iterations need in one epoch.
The role of training dataset in deep CNN is the source of features. It works as image library to track features based on it the model can decide and predict.

An imperative thing in back-propagation process is getting Global Loss Minimum which mean square error is equal to the lowest error rate. After setting the factors, which cause to get the best performance, the model can recognize the images according to the classes. Some factors increase performance of intelligent methods, such as; dataset, optimizer, number of epochs, batch size, learning rate, in addition to some other pre-processes operations as; resizing images dataset to a desired size which cause to accelerate the process, and data augmentation. In the processes of classification and detection, datasets can use as resource to deep learning methods especially for the proposed method. The proposed method capable to expand and manipulate many complex problems relate to computer vision, which can manipulate higher image quality compare with some of other methods. In addition, this method can involve many image types relating to COVID-19 pneumonia to diagnose and follow-up the COVID-19 pneumonia progression situations, also evaluate the stages. It can use as criterion to classify the patients, which aid the medication process. This process implemented on MATLAB with best accuracy, which is $99.37 \%$ according to the collected dataset, as shown in Figure 4 and it fixed to set on optimum situation.

\section{Conclusions}

The study reveals that auto-diagnoses of the COVID-19 are too necessary. CT images of the classes are too different from each other. Some factors have the most impacts to increase accuracy. The factors consist of the dataset, deep CNN method, training options. The dataset represents as the feature bank. Deep CNN method involves feature extraction and classification processes together and necessary layers until the most significant features arrive to the classification layers to vote. Training options, 
which include the optimizer and number of epochs and learning rate. The performance of Deep CNN in terms of its feature learning accuracy is highly dependent on the number of utilized epochs, which set to 12 in the present study. The whole autodiagnoses system runtime did not subtend more than 2 minutes, representing real-time diagnosis. Accurate diagnosis of COVID19 pneumonia CT images is highly useful in follow-up and diagnosis, in turn rising the patient's lifetime. In this regard, deep $\mathrm{CNN}$ is one of the most significant and effective models that in automated COVID-19 pneumonia diagnosis, with the classification capacity of hundreds of images per second.

\section{References}

[1] Y. Song, S. Zheng, L. Li, X. Zhang, X. Zhang, Z. Huang, J. Chen, H. Zhao, Y. Jie, R. Wang, Y. Chong, J. Shen,Y. Yang, "Deep learning Enables Accurate Diagnosis of Novel Coronavirus (COVID-19) with CT images," IEEE TRANSACTIONS ON MEDICAL IMAGING, 1-1, 2020, DOI 10.1109/TMI.2020.2995965.

[2] M. Hosseiny, S. Kooraki, A. Gholamrezanezhad, S. Reddy and L. Myers, "Radiology Perspective of Coronavirus Disease 2019 (COVID-19): Lessons From Severe Acute Respiratory Syndrome and Middle East Respiratory Syndrome," American Journal of Roentgenology,215,1-5, 2020, DOI: 10.2214/AJR.20.22969. Epub 2020 Feb 28.

[3] L. Li, L. Qin, Z. Xu, Y. Yin, X. Wang, B. Kong, J. Bai, Y. Lu, Zhenghan, "Artificial Intelligence Distinguishes COVID-19 from Community Acquired Pneumonia on Chest CT," Radiology,200905, 2020, DOI: 10.1148/radiol.2020200905.

[4] A. Srivastava, S. Sengupta, SJ. Kang, K. Kant, M. khan, S. Asad Ali, S. R. Moore, B. C. Amadi, P. Kelly, S. Syed, and D. E. Brown, "Deep Learning for Detecting Diseases in Gastrointestinal Biopsy Images," in 2019 Systems and Information Engineering Design Symposium (SIEDS), Charlottesville, VA, USA, 26-26 April 2019, DOI 10.1109/SIEDS.2019.8735619.

[5] Mpesiana, I. D. Apostolopoulos and A. Tzani, "Covid-19: automatic detection from X-ray images utilizing transfer learning with convolutional neural networks," Physical and Engineering Sciences in Medicine, 2020, DOI: https://doi.org/10.1007/s13246-020-00865-4

[6] W. Zhao, Z. Zhong, X. Xie, Q. Yu, J. Liu , "CT Scans of Patients with 2019 Novel Coronavirus (COVID-19) Pneumonia," Theranostics, 10(10), 4606-4613, 2020, doi:10.7150/thno.45016.

[7] X. Xu, X. Jiang, C. Ma, P. Du, X. Li, S. Lv, L. Yu, Q. Ni, Y. Chen, J. Su, G. Lang, Y. Li, H. Zhao, J. Liu, K. Xu, L. Ruan, J. Sheng, Y. Qiu, W. Wu, T. Liang, L. Li, "Deep Learning System to Screen Coronavirus Disease 2019," arxiv, 1-29, 2020, DOI: 10.1016/j.eng.2020.04.010.

[8] Y. Xu, "Dynamic profile of severe or critical COVID-19 cases", medRxiv, 2020, DOI: https://doi.org/10.1101/2020.03.18.20038513.

[9] K. C. Santosh, "AI-Driven Tools for Coronavirus Outbreak: Need of Active Learning and Cross-Population Train/Test Models on Multitudinal/Multimodal Data," Journal of Medical Systems, 44(93), 2020.

[10] H. Shin, H. R. Roth, M. Gao, L. Lu, Z. Xu, I. Nogues, J. Yao, D. Mollura, R. M. Summers, "Deep Convolutional Neural Networks for ComputerAided Detection: CNN Architectures, Dataset Characteristics and Transfer Learning," IEEE Transactions on Medical Imaging, 35(5), 1285-1298, 2016.

[11] Raja, P. M. Krishnammal and S. Selvakumar, "Convolutional Neural Network based Image Classification and Detection of Abnormalities in MRI Brain Images," in 2019 International Conference on Communication and Signal Processing (ICCSP), Chennai, India, 4-6 April 2019, DOI: 10.1109/ICCSP.2019.8697915

[12] G. Litjens, T. Kooi, B. E. Bejnordi, A. A. A. Setio, F. Ciompi, M. Ghafoorian, J. A.W.M.V. Laak, B. V. Ginneken, C. S. Diagnostic, "A survey on deep learning in medical image analysis," Medical Image Analysis, 42, 60-88, 2017, DOI: 10.1016/j.media. 2017. 07005.

[13] A. Vedaldi, and K. Lenc of Oxford, "Convolutional Neural Networks for MATLAB,” ACM, 15(10), 2015, DOI: 10.1145/3210241.3210250.
[14] Shan, Z. J. Cheng and Jing, "2019 Novel coronavirus: where we are and what we know," Infection, 155-163, 48, 2020, DOI: 10.1007/s15010-02001401-y.

[15] J. Liu , "Artificial Intelligence Assisted Radiology Technologies Aid COVID-19 Fight in China," itn IMAGING TECHNOLOGY NEWS, 2020.

[16] L. Huang, R. Han, T. Ai, P. Yu, H. Kang, Q. Tao, L. Xia, "Serial Quantitative Chest CT Assessment of COVID-19: Deep-Learning Approach,"Radiology: Cardiothoracic Imaging, 2(2), 2020, DOI:10.1148/ryct.2020200075.

[17] D. Varshni, K. Thakral, L. Agarwal, R. Nijhawan, A. Mittal, "Pneumonia Detection Using CNN based Feature Extraction," in 2019 IEEE International Conference on Electrical, Computer and Communication Technologies (ICECCT), Coimbatore, India ,20-22 February 2019, DOI: 10.1109/ICECCT.2019.8869364.

[18] C. Zheng, X. Deng, Q. Fu, Q. Zhou, J. Feng, H. Ma, W. Liu, X. Wang, "Deep Learning-based Detection for COVID-19 from Chest CT using Weak Label," IEEE TRANSACTIONS ON MEDICAL IMAGING,1-1, 2020, DOI: 10.1109/TMI.2020.2995965.

[19] A. Narin, C. Kaya, Z. Pamuk, "Automatic Detection of Coronavirus Disease (COVID-19) Using X-ray Images and Deep Convolutional Neural Networks," arXiv, 2020.

[20] M. Loey, F. Smarandache, N. E. M. Khalifa, "Within the Lack of COVID19 Benchmark Dataset: A Novel GAN with Deep Transfer Learning for Corona-virus Detection in Chest X-ray Images," Symmetry,12(4), 2020 doi:_10.3390/sym12040651. 